\section{Helping Elementary Teachers Build Mathematical Generality into Curriculum and Instruction ${ }^{1}$}

\author{
Maria L. Blanton and James J. Kaput \\ Dartmouth, MA (USA)
}

\begin{abstract}
This article explores how elementary teachers can use functional thinking to build algebraic reasoning into curriculum and instruction. In particular, we examine how children think about functions and how instructional materials and school activities can be extended to support students' functional thinking. Data are taken from a five-year research and professional development project conducted in an urban school district as well as from a graduate course for elementary teachers taught by the first author. We propose that elementary grades mathematics should, from the start of formal schooling, extend beyond the fairly common focus on recursive patterning to include curriculum and instruction that deliberately attends to how two or more quantities vary in relation to each other. We discuss how teachers can transform and extend their current resources so that arithmetic content can become opportunities for pattern building, conjecturing, generalizing, and justifying mathematical relationships between quantities and how teachers might embed this mathematics within the kinds of sociomathematical norms that help children build mathematical generality.
\end{abstract}

Kurzreferat: Der Beitrag untersucht, wie Grundschullehrerinnen und -lehrer funktionales Denken einsetzen können, um algebraisches Argumentieren in Curriculum und Unterricht zu integrieren. Insbesondere wird untersucht, wie Kinder über Funktionen und wie Unterrichtsmaterial und Unterrichtsaktivitäten ausgeweitet werden können, um das funktionale Denken der Schülerinnen und Schüler zu fördern.

Dabei stützt sich der Beitrag auf Daten aus einer Fünf-JahresStudie, die im Rahmen eines Forschungs- und Fortbildungsprojekts in einem städtischen Schulbezirk sowie in von dem erstgenannten Autor durchgeführten Universitätsveranstaltungen für Grundschullehrerinnen und -lehrer durchgeführt wurde. Es wird vorgeschlagen, dass der Mathematikunterricht bereits ab seinem Beginn über die weithin übliche Thematisierung rekursiver Muster hinausgehen und curriculare Aspekte berücksichtigen sollte, die bewusst die Veränderung zweier oder mehrerer Größen thematisieren. Es werden Vorschläge entwickelt, wie Lehrpersonen ihre üblichen Ressourcen erweitern können, so dass arithmetische Inhalte Gelegenheit bieten für die Entwicklung von Mustern, Vermutungen, Verallgemeinerungen und Begründungen von mathematischen Beziehungen zwischen Größen. Des Weiteren sollen die Vorschläge Lehrpersonen aufzeigen, wie sie diese Art von Mathematik in sozio-mathematische Normen einbetten können, um den Kindern zu helfen, mathematische Verallgemeinerungen zu entwickeln.

\section{ZDM-Classification: D40, H20}

\footnotetext{
1 The research reported here was supported in part by a grant from the U.S. Department of Education, Office of Educational Research and Improvement to the National Center for Improving Student Learning and Achievement in Mathematics and Science (R305A600007-98). The opinions expressed herein do not necessarily reflect the position, policy, or endorsement of supporting agencies.
}

\section{Introduction}

Current research is redefining what we understand about the kind of mathematics that young children can and should learn (National Research Council [NRC] 2001). Consider the following Towers of Cubes problem (see Fig. 1) taken from the National Council of Teachers of Mathematics [NCTM] Principles and Standards for School Mathematics (2000, p.160):

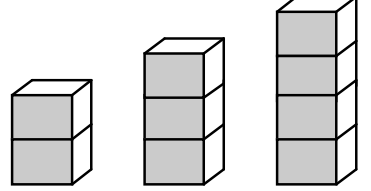

What is the surface area of each tower of cubes (include the bottom)? As the tower gets taller, how does the surface area change? What is the surface area of a tower with fifty cubes?

Figure 1: Towers of Cubes.

In the not so distant past, such a problem was mostly absent from typical elementary school ${ }^{2}$ curricula and instruction in the United States. While it might have appeared as an enrichment task, it was likely marginalized by the press towards computational skills (Thompson, Philipp, Thompson, \& Boyd 1994) and procedures that children were (and are) compelled to memorize as a signal of their readiness for higher mathematical thinking. Or, it might have appeared in an abbreviated, arithmetic form as "What is the surface area of a tower built of 3 cubes?" However, algebraic reasoning as an activity of generalizing mathematical ideas, using literal symbolic representations, and representing functional relationships, all implicit in this task, is no longer reserved for secondary grades and beyond, but is an increasingly common thread in the fabric of ideas that constitute mathematical thinking at the elementary grades.

\subsection{The challenge of curriculum and instruction}

Simply put, young children today need to learn a different kind of mathematics than their parents learned. Some would argue that they need to be "algebra ready". But what experiences make them ready for algebra, and for what kind of algebra are they being made ready? Romberg and Kaput (1999) maintain that understanding the increasingly complex mathematics of the $21^{\text {st }}$ century will require children to have a type of elementary school experience that goes beyond arithmetic and computational fluency to attend to the deeper underlying structure of mathematics. It will require experiences that help children learn to recognize and articulate mathematical structure and relationships and to use these insights of mathematical reasoning as objects for mathematical reasoning (Blanton to appear). This type of elementary school experience has come to be embodied in what many refer to as early algebra ${ }^{3}$, and because its

\footnotetext{
2 Elementary school refers here to grades PreK-5.

While there are multiple perspectives on early algebra, Lins and Kaput (2004) describe a general agreement among scholars that it involves "acts of deliberate generalization and expression of generality... [and] reasoning based on the forms
} 
underlying purpose is to deepen children's understanding of the structural form and generality of mathematics and not just provide isolated experiences in computation, scholars increasingly agree that it is the avenue through which young children can become mathematically successful in later grades. Thus, our perspective on "algebra readiness" is that experiences in building and expressing mathematical generalizations-for us, the heart of algebra and algebraic thinking - should be a seamless process that begins at the start of formal schooling, not content for later grades for which elementary school children are "made ready".

But changing the mathematics elementary school children learn is only part of the solution. As Blanton and Kaput note, "most elementary teachers have little experience with the kinds of algebraic thinking that need to become the norm in schools and, instead, are often products of the type of school mathematics instruction that we need to replace" (to appear). However, these very teachers are central to changing children's school mathematical experiences. Moreover, the instructional materials in most elementary schools today are basal texts, and even newer, standards-based materials are just beginning to incorporate systematic approaches to the development of algebraic reasoning (Kaput \& Blanton to appear). These constraints represent the challenge of building algebraic reasoning into curriculum and instruction.

\subsection{Functional thinking as a route to algebraic thinking}

There are two issues implicit in the above discussion that this article aims to address: (1) how opportunities for algebraic reasoning can be integrated into the elementary grades to prepare students for more powerful mathematics in later years, and (2) how elementary teachers can transform their own resources and instruction in ways that effect (1).

Early algebra can occur in several interrelated forms in the classroom ${ }^{4}$. We focus here on functional thinking as a strand by which teachers can build generality into their curriculum and instruction. We broadly conceptualize functional thinking to incorporate building and generalizing patterns and relationships using diverse linguistic and representational tools and treating generalized relationships, or functions, that result as mathematical objects useful in their own right. In addition, we use here three modes of analyzing patterns and relationships, outlined by Smith (to appear), as a framework to discuss the kinds of functional thinking found in classroom data: (1) recursive patterning involves finding variation within a sequence of values; (2) covariational thinking is based on analyzing how two

of syntactically guided actions on those expressions".

4 Kaput (1999) characterizes algebraic thinking as consisting of several inter-related forms: (a) the use of arithmetic as a domain for expressing and formalizing generalizations; (b) generalizing numerical patterns to describe functional relationships; (c) modeling as a domain for expressing and formalizing generalizations; and (d) generalizing about mathematical systems abstracted from computations and relations. quantities vary simultaneously and in keeping that change as an explicit, dynamic part of a function's description (e.g., "as $x$ increases by one, $y$ increases by three") (Confrey \& Smith 1991); and (3) a correspondence relationship is based on identifying a correlation between variables (e.g., " $y$ is 3 times $x$ plus 2 ").

In what follows, we draw on data from a five-year research and professional development project (Kaput \& Blanton to appear) and a subsequent graduate course for elementary teachers taught by the first author to examine how children think about functional relationships, its mathematical implications for later grades, and how instructional materials and school activities can be deepened and extended to support the development of functional thinking. First, we briefly describe the project and its participants.

\subsection{The GEAAR Project}

The Generalizing to Extend Arithmetic to Algebraic Reasoning [GEAAR] Project was conducted in one of the lowest achieving school districts in the state based on state-wide, standardized assessments. The district is located in an economically and educationally constrained community, where the median yearly income is $\$ 25,000$, the per capita yearly income is $\$ 13,000$, and approximately $50 \%$ of the community's inhabitants did not complete high school.

GEAAR was designed to develop teachers' abilities to identify and strategically build upon students' attempts to reason algebraically and to use existing and supplemental instructional resources to engineer classroom activities to support this. Its format was based on engaging teachers in (1) collectively solving authentic algebraic reasoning tasks in cross-grade teams, (2) adapting these tasks for use in their own classrooms, (3) implementing the tasks with their students, and (4) sharing with colleagues their analyses of students' thinking about the tasks. During biweekly meetings with the authors, teachers were encouraged to think about whether a culture of inquiry and argumentation was developing in their classrooms, the nature of the differences across classrooms in how students thought about mathematical ideas, and how the teachers themselves perceived the evolution of their practice.

After the first year of the project, teacher participants were recruited to lead, in pairs, groups of new teacher participants in year-long seminars modeled after GEAAR. In many cases, the newly formed groups were based on whole school participation. Throughout the remainder of GEAAR, we met with teacher leaders approximately biweekly in order to oversee the expansion of GEAAR throughout the district. It was through these types of long-term interactions with teachers that we were able to address how they might change their curriculum (based on a typical, mainstream basal series) and instruction.

The course taught by the first author extended the findings of GEAAR and had, as its participants, teachers from the GEAAR project district as well as other surrounding (and, in some cases, more academically successful) districts.

Accounts of this work include a detailed case study of 
a classroom practice that promotes algebraic thinking (Blanton \& Kaput to appear), the kinds of curricular, programmatic, and institutional designs that support it (Blanton \& Kaput 2001, 2003, to appear; Kaput \& Blanton to appear), and its impact on student achievement (Kaput \& Blanton 2001).

\section{Functional thinking in the elementary grades}

The idea of function has, for over a century, been regarded by mathematicians as a powerful, unifying idea in mathematics that is worthy of a central place in the curriculum (Freudenthal 1982; Hamley 1934; Schwartz 1990). Indeed, the idea can be traced back to Leibniz (Boyer 1946). However, until very recently, the idea of function has been treated largely as something to be learned in high school algebra, or, in the case of recent standards-based curricula, middle school mathematics. The perspective taken here is that functions should be treated longitudinally and in its full richness beginning in early elementary school (Smith 2003).

But what capacity do young children have for functional thinking? Even though elementary school mathematics has more recently included recursive patterning, it has not attended pervasively to covariation or correspondence in functional thinking, especially in grades PreK-2. For instance, even NCTM (2000) suggests that, as late as fourth-grade, students might find a recursive pattern in the Towers of Cubes problem (see Fig. 1), and not until fifth-grade would they develop a correspondence relationship. Can elementary students, in fact, make the conceptual shift from simple recursive patterning to account for simultaneous changes in two or more variables? Moreover, at what grades can they do this? And can they, or in what ways can they, symbolize and operate on covariational or correspondence relationships in data?

\subsection{Children's capacity for functional thinking}

Current research challenges the developmental constraints traditionally placed on young learners and their capacity for functional thinking. For example, researchers have found that elementary school children can develop and use a variety of representational tools to reason about functions, they can describe in words and symbols recursive, covarying, and correspondence relationships in data, and they can use symbolic language to model and solve equations with unknown quantities (e.g., Blanton to appear; Brizuela \& Schliemann 2003; Brizuela, Carraher, \& Schliemann 2000; Carraher, Schliemann, \& Brizuela 2000; Kaput \& Blanton to appear; Schliemann \& Carraher 2002; Schliemann, Carraher, \& Brizuela 2001).

While much of this research focuses on functional thinking in grades 3-5, we have found that students are not only capable of deeper functional analysis than previously thought, but that the origins of these ideas appear at grades earlier than typically expected. In particular, we have found that the types of representations students use, the progression of mathematical language in their descriptions of functional relationships, the ways students track and organize data, the mathematical operations they use to interpret functional relationships, and how they express covariation and correspondence among quantities, can be scaffolded in instruction beginning with the very earliest grades, at the start of formal schooling (Blanton \& Kaput 2004).

The following discussion draws on our research data to elaborate these capacities in children's functional thinking across grades PreK-5. We note that the data included here are intended to convey existence proofs of what is possible in children's thinking; our goal is not to examine the regularity with which functional thinking occurred in instruction.

\subsubsection{The development of representational infrastructure}

Research, including early algebra research, suggests that students' flexibility with multiple representations both reflects and promotes deeper mathematical insights (Behr, Lesh, Post, \& Silver 1983; Brizuela \& Earnest to appear; Goldin \& Shteingold 2001). Brizuela and Earnest note that "the connections between different representations help to resolve some of the ambiguity of isolated representations, [so] in order for concepts to be fully developed, children will need to represent them in various ways".

We found that teachers across grades PreK-5 were able to scaffold students' use of tables, graphs, pictures, words and symbols in gradually more sophisticated ways in order to make sense of data and interpret functional relationships (Blanton \& Kaput 2004). For example, while students in grades PreK-1 relied on counting visible

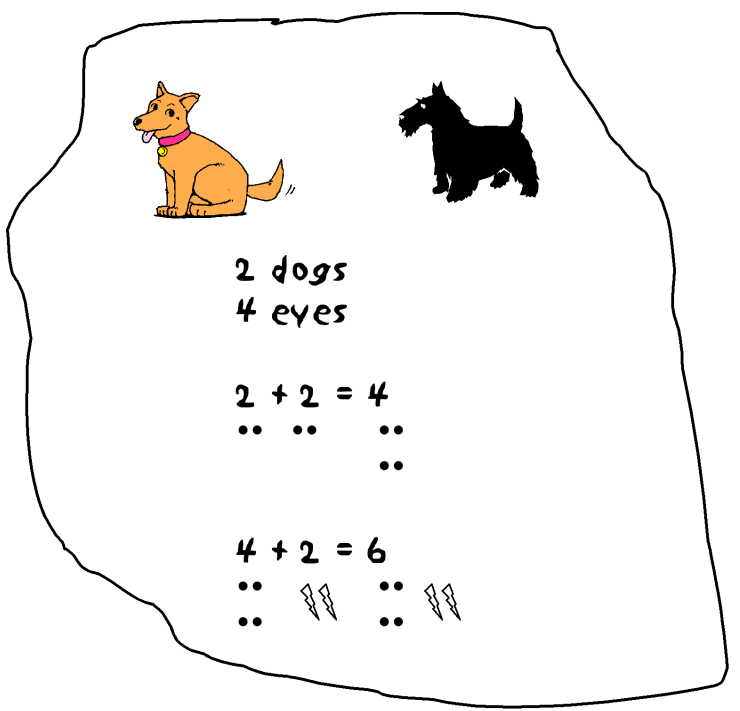

Figure 2: Kindergarten Students' Representation for the Numbers of Eyes and Eyes and Tails for Two Dogs.

objects and registering their counts through inscriptions in $\mathrm{t}$-charts ${ }^{5}$ or through dots and hatch marks for eyes and tails (see Fig. 2), by second and third grade, students could routinely operate on data that had no iconic or tangible counterpart (e.g., tracking the number of eyes on ten dogs without pictures or physical objects). Moreover,

\footnotetext{
5 T-charts are teacher-termed function tables with a column of data for the independent variable followed by a column of data for the dependent variable.
} 
while grades PreK-1 teachers typically led students in developing $\mathrm{t}$-charts to organize their data, the responsibility for this began to shift to students during first grade. By second and third grades, students were able to use this tool transparently, as a mathematical object, in thinking about data.

The t-chart became an important structure in students' mathematical reasoning. In the earlier grades (PreK-1), it provided a context to re-represent marks with numerals as children worked on the arithmetic of correspondence between quantity and numeral. But its introduction in these grades as a tool for organizing covarying data also initiated its transformation from opaque to transparent object (Kaput 1995) in children's functional thinking as a representation that one could "look through" to "see" new relationships. We maintain that introducing such representational tools from the start of formal schooling can help spread the cognitive load across grades in a way that allows second and third grade students to focus on more difficult tasks such as symbolizing correspondence and covariational relationships.

The following teacher narrative illustrates that, by third-grade, students found tools such as t-charts critical in reasoning mathematically. The third-grade teacher who authored the narrative, Mrs. Gardiner, had designed a task in which students were to find the number of body parts a growing snake would have on day 10 and on day $n$, where each triangle equaled a body part. She drew the growing snake on the board for Days 1, 2, and 3 (see Fig. $3)$.

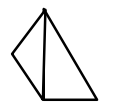

Day 1

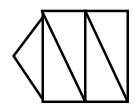

Day 2

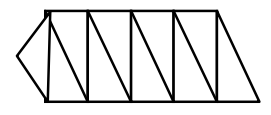

Day 3
Figure 3: The Growing Snake.

\begin{abstract}
"The class worked on this problem for approximately 10 minutes. All organized their data with a t-chart. When I pulled the group together to discuss the problem, it was Karlie ${ }^{6}$ who had her hand waving hard.... Karlie usually just sits and listens during math time, so her enthusiasm was very special. I called on her right away. 'I know that on day 10 the snake will have 101 body parts and I know that on day $n$ the snake will have $n \square n+1$. I know this because I used my t-chart and I looked for the relationship between $n$ and body parts. This is the first time I saw the pattern, so please tell me I'm right!' she said excitedly.... The class had all come to pretty much the same answer".
\end{abstract}

This suggests to us that the t-chart helped structure Karlie's thinking about relationships between quantities. Unlike grades PreK and K, where students used t-charts opaquely as a storage system for numbers and were not yet able to attend to the meanings embedded in how data were positioned in the chart, the t-chart became the object, or tool, by which Karlie could compare data and find relationships. She was able to attend to how numbers were located in the chart and see through it to the relationships it made available to her. In this sense, we maintain that the t-chart had become transparent in how

\footnotetext{
${ }^{6}$ All student names are pseudonyms
}

she used it to think about functions. Our point is that critical instruction in the earlier grades (PreK-1) can initiate the transition of representational tools from opaque to transparent objects in children's thinking so that children are able to shift their attention to more complex tasks in later elementary grades and beyond. This is exactly how mathematics has grown in power historically, as new representation systems were developed (including that of algebra itself) to increase the power of human thinking.

\subsubsection{The development of students' symbol sense}

One particularly vital aspect of early algebra is the transition from natural language to symbolic notational systems. If one's perspective is that development precedes learning, then the use of symbols as variables in elementary grades is, perhaps, not without controversy. However, we take the view here that learning promotes development and that it entails a pseudo-conceptual stage of concept formation in students' development of symbol sense. In describing the development of higher mental functioning in children, Vygotsky (1962) identified the notion of a pseudo-concept as an essential bridge in children's thinking to the final stage of concept formation. While the pseudo-concept a child possesses is phenotypically equivalent to that of an adult, it is psychologically different. As a result, the child is able to "operate with [the concept], to practice conceptual thinking, before he is clearly aware of the nature of these operations". (p. 69) This suggests that learning to think mathematically involves the acquisition of notational tools that are within the child's zone of proximal development, but not entirely owned by the child. In essence, it involves students' transition from an opaque to transparent use of symbols. Moreover, the dialectic between thought and language in learning (Vygotsky 1962) implies that symbolic notational systems are more fully conceptually formed in children's thinking as a result of children's interaction with them in meaningful contexts. In short, children can develop symbol sense as they have opportunity to use symbolic notation in meaningful ways (see also Brizuela, Carraher, \& Schliemann 2000).

We have found that, when curriculum and instruction provide opportunity for thinking about functional relationships, students can transition linguistically from iconic and natural language registers at grades PreK-1 to symbolic notational systems by grade 3 (Blanton \& Kaput 2004). We include the following teacher narrative to illustrate third-grade students using symbolic notation to think about the number of circle-shaped body parts on a growing caterpillar. In this vignette, Mrs. Gardiner has just described the Growing Caterpillar task to her students ${ }^{7}$.

"I showed my students my caterpillar example and all I wanted them to see was how I developed the problem. I had no idea that

\footnotetext{
${ }^{7}$ Growing Caterpillar was similar to Growing Snake except for the shape of the body parts. The growth rates for the snake and caterpillar were the same. Mrs. Gardiner had given Growing Caterpillar to students two weeks prior to Growing Snake.
} 
they would begin to solve the problem. I couldn't stop them. There were hands going up all over the place. Everyone wanted to tell me the pattern they saw when they looked at the growth of the caterpillar. I said, 'Guys, I haven't even asked you the question yet'. 'But I see the pattern, Mrs. Gardiner', yelled Jak. 'Okay, what do you think the pattern is?' I asked. 'I think it is $x$ times 2 plus one', he said. 'How many of you agree with Jak?' I questioned. 'I don't know. I have to do a t-chart', explained Meg. 'Well, then let's do that together on the board', I said. With the students' help, we drew the following t-chart on the board (see Fig. 4):

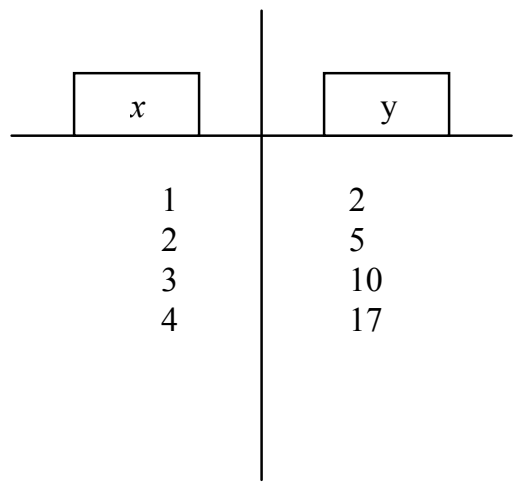

Figure 4: T-chart for Growing Caterpillar.

'Now that we have that on the board, I don't agree with Jak', said Meg. 'Why is that Meg?' I asked. 'Because if it was $x$ times 2 plus 1 , then $x$ would be one and $y$ would be three. And, it's not. It's $x=1$ and $y=2$ ', she explained.... [If $x$ equaled 1, then by Jak's formula, $y$ would be $2(1)+1$, or 3 , not 2 , as the tchart indicated.]' The class struggled with the pattern for a long time. Then Shane saw a pattern that I had not seen. He came up to the t-chart on the board and with a red marker highlighted the pattern. It looked like this (see Fig. 5):

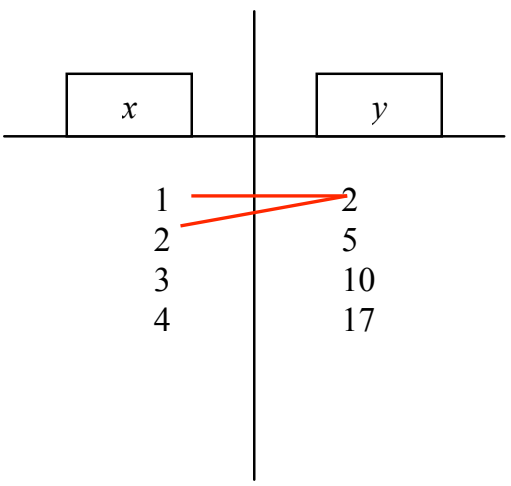

Figure 5: Shawn's Pattern for Growing Caterpillar Task.

So, what Shane was saying is that if you add $1+2+2$, it equals 5. If you then add $2+5+3$, it equals 10 . This...didn't help him find the formula, but it did help Joe! 'I see it, I know the formula!' Joe cried out. 'Well, what is it?' I prodded. 'It's $x \square x+1=y$ ', he said. At that moment, a loud group of 'Oh yeah's' could be heard in the room.... I asked everyone why this was algebra. I think Jak put it best. He said, 'Because we have people looking for patterns and relationships and we have them developing a formula"'.

There are several points with respect to students' use of symbols that bear mentioning here. First, before any data were publicly recorded and without any prompting from the teacher, Jak proposed a symbolic relationship between an arbitrary day, $x$, and the number of caterpillar body parts. His spontaneous use of symbols conveys the generality with which he was beginning to think about functional relationships. Second, Meg was beginning to reason transparently with the $\mathrm{t}$-chart and the symbolic relationship conjectured by Jak in order to refute his idea ("Now that we have that on the board, I don't agree with Jak"). That is, implicit in her refutation was her reasoning with both the meaning embedded in the structure of the tchart, including the unique roles of dependent and independent variables, as well as the symbolic notation ("Because if it was $x$ times 2 plus 1 , then $x$ would be one and $y$ would be three. And, it's not. It's $x=1$ and $y=2$ "). Meg's emerging transparent use of symbolic notation is further indicated by her treatment of the expression ' $x$ times 2 plus 1 ' and the dependent variable, $y$, as equivalent quantities.

Because the elementary grades often incorporate meaningful imagery and concrete experiences to support conceptual development, they, more so than secondary grades, can provide a rich, inquiry-based atmosphere for introducing symbolic notation. Thus, as with the development of representational infrastructure, we maintain that instruction should begin to scaffold students' thinking toward symbolic notation from the start of formal schooling so that students can transition from an opaque to transparent use of symbols throughout the elementary grades. Ultimately, elementary students who have learned to reason symbolically in meaningful ways will be much better prepared for the abstractions of more advanced mathematical thinking in later grades.

\subsubsection{The emergence of thinking about covariational and correspondence relationships}

We have found it particularly compelling that, as early as kindergarten, children can think about how quantities covary and, as early as first grade, can describe how quantities correspond (Blanton \& Kaput 2004). In particular, in a task in which students were asked to describe the total number of eyes or the total number of eyes and tails for an increasing number of dogs, one kindergarten class described an additive covariational relationship between the number of eyes and dogs as "every time we add one more dog we get two more eyes". In first and second grade, students identified a multiplicative relationship of "doubles" and "triples" between the number of eyes and the number of eyes and tails, respectively, for an increasing number of dogs. The observation that the pattern "doubles" or "triples" suggests that students could attend to how quantities corresponded. For example, some quantity needed to be doubled to get the total amount of eyes. Since data representing the total number of eyes (i.e., $2,4,6,8 \ldots$ ) were not doubled to get subsequent quantities of dog eyes (4 doubled does not yield the next value of 6; 6 doubled does not yield the next value of 8 ), this suggests that students were not looking for a recursive pattern such "add 2 every time" or "count by 2 's", but a relationship between two quantities.

We recognize that some children might be responding 
to a known relationship without fully understanding its functional aspect. "Doubles", for example, is not uncommon in the vocabulary of early grades mathematics, and to say "it doubles" does not necessarily indicate a full conceptual understanding of correspondence or covariation. For some children, "doubles" could be code for a pattern recognized as adding by two's. However, these situations can prompt discussions that scaffold students' thinking about relationships between data, not just recursive patterning. As the Growing Snake and Growing Caterpillar excerpts suggest, by third grade students can attend to how quantities co-vary and, moreover, symbolize relationships as a functional correspondence (e.g., "It's $x \square x+1=y$ "). Thus, although the data on dog eyes and tails illustrate a simple mathematical relationship for which some children used only natural language to describe covariational and correspondence relationships, we think this represents the critical kinds of experiences that children need in the earlier elementary grades in order to leverage deeper, more complex functional thinking in later elementary grades.

\subsubsection{Implications of children's functional thinking for later grades}

The preceding discussion underscores how early algebra, and functional thinking in particular, can nurture the development of students' mathematical thinking in later grades. To begin with, it can help children build critical representational and linguistic tools for analyzing, describing and symbolizing patterns and relationships. Moreover, if teachers scaffold these ideas from the start of formal schooling, these experiences can provide a continuum of mathematical development whereby opaque symbols and tools can be transformed into transparent objects of functional thinking. T-charts and graphs become not just visual configurations, but structures embedded with meaning about relationships; symbols are no longer meaningless abstract inscriptions, but tools by which broader ideas that can be mediated and communicated. Moreover, the elementary grades, because of its inclination towards concrete, tactile, and visual experiences in learning, can bridge the expression of mathematical ideas from natural, everyday language to symbolic notational systems in meaningful ways. For example, students in secondary grades are often given, $a$ priori, a symbolic generalization about the commutative property of addition for real numbers $a$ and $b(a+b=b$ $+a$ ). In contrast, early algebra entails exploring this property through operations on particular numbers, then generalizing the property using everyday or symbolic language systems, where the symbolizing develops as a valid linguistic form of expression through children's interactions with number and operation (Carpenter, Franke, Levi 2003).

All of these experiences-the development of representational and linguistic tools, the transformation of mathematical structures and symbols from opaque to transparent objects, and the integration of concrete, tactile, and visual experiences to support the development of mathematics with understanding - coalesce to build mathematical thinkers for whom abstract ideas are rooted in meaningful, concrete events. As a result, we argue that children for whom functional thinking is a routine part of mathematics in the elementary grades are better prepared than those who spend the first six or seven years of formal schooling fine-tuning arithmetic skills, procedures and facts.

\section{Integrating functional thinking into curriculum and instruction}

While much more could be said about children's capacity for functional thinking, our point thus far is that young children can identify and express functional relationships in progressively more symbolic ways and that instruction in the elementary grades that nurtures this kind of thinking can support students' mathematical thinking in later grades. Although this suggests a mandate for change in elementary school curricula, our reality is often working with teachers who have limited resources that, more often than not, focus on the development of children's arithmetic thinking. Moreover, curricular innovations alone, without the development of teachers' instructional and mathematical knowledge on how to build children's functional thinking, are not sufficient to produce real change in children's mathematical thinking. Smith (2003) notes that "elementary school teachers may create rich classroom experiences around patterns, yet not have a sense of how this topic ties into the ongoing mathematical development of their students, much less into the topic of functions". (p. 136) To address this, our early algebra work with teachers has involved three connected dimensions of change: (1) transforming teachers' instructional resource base, (2) using children's thinking to leverage teacher learning, and (3) creating classroom culture and practice to support algebraic thinking. In what follows, we address each of these and how they support the integration of functional thinking into curriculum and instruction.

\subsection{Transforming teachers' resource base to support students' functional thinking}

In spite of limited resources or the lack of materials that integrate functional thinking in viable ways, elementary teachers can transform their existing instructional resource base to include the exploration of covariational and correspondence relationships. Our approach with teachers is to help them deliberately transform singlenumerical-answer arithmetic problems to opportunities for pattern building, conjecturing, generalizing, and justifying mathematical relationships by varying the given parameters of a problem (Blanton \& Kaput 2003). This is easily done with tasks such as the Telephone Problem, which might typically be posed as an arithmetic task with a single numerical answer:

How many telephone calls could be made among 5 friends if each person spoke with each friend exactly once on the telephone?

Stated this way, students simply need to compute a sum, although they might first draw a picture or diagram to keep track of the phone calls. Functional thinking can be introduced into the task by varying the number of friends in the group: 
How many telephone calls would there be if there were 6 friends? Seven friends? Eight friends? Twenty friends? One hundred friends? Organize your data in a table. Describe any relationship you see between the number of phone calls and the number of friends in the group. How many phone calls would there be for $n$ friends?

The tasks included here (e.g., Growing Snake, Growing Caterpillar, Towers of Cubes) are examples of this type of transformation; all are derived from single-numericalanswer tasks. For example, Towers of Cubes can be seen as an extension of the arithmetic problem "What is the surface area of a tower built of 3 one-inch cubes?" Similarly, Growing Snake can be seen as an extension of an arithmetic task in which students count the total number of body parts for a particular snake.

\subsubsection{Varying task parameters introduces algebraic thinking into the curriculum}

But how does this transformation lead to algebraic thinking or, specifically, functional thinking? First, varying a problem parameter enables students to generate a set of data that has a mathematical relationship, and using sufficiently large quantities for that parameter leads to the algebraic use of number. For example, in the Telephone Problem, finding the number of phone calls for a group whose size is large enough so that children cannot (or would not want to) model the problem and write down a corresponding sum to compute requires children to think about the structure in the numbers and how the numbers of phone calls for the various groups are related to the number of people in the group. From their analysis, children can identify a recursive pattern or conjecture a covariational or correspondence relationship between the total numbers of phone calls and the variations in the parameter that produces them. Moreover, depending on the grade and skill of the student, the teacher can scaffold students in describing their conjectures with symbolic notation. Children can then develop justifications for whether or not their conjectured relationships and patterns hold true. Finally, the mathematical generalizations that result, while important results in and of themselves, can become objects of mathematical reasoning as students become more sophisticated algebraic thinkers (Blanton \& Kaput 2000). None of these processes occur if tasks remain purely arithmetic in scope.

As we describe elsewhere, our approach "recasts elementary mathematics in a profound way, not by ignoring its computational agenda, but by enlarging the agenda in ways that include the old in new forms that deliberately contextualize, deepen, and leverage the learning of basic skills and number sense by integrating them into the formulation of deeper mathematical understandings" (Kaput \& Blanton to appear). In essence, a powerful result of transforming arithmetic tasks in this way is that children are doing many important things all at once, including building number sense, practicing number facts, building and recognizing patterns to model situations, and so forth (Blanton \& Kaput 2003). In fact, this genre of tasks can provide large amounts of computational practice in a context that intrigues students and that avoids the mindlessness of numerical worksheets.

\subsubsection{Transforming the curriculum empowers teachers}

Moreover, we have found that when teachers transform their own instructional resource base so that arithmetic tasks are extended to include opportunities for establishing and expressing mathematical generalizations, they are able to transcend constraints imposed by their existing school culture, such as limited or inadequate resources, and to see algebraic thinking as a fluid domain of thinking which permeates all of mathematics, not as a set of tasks or a prescribed curriculum. Thus, what we advocate, more so than an "early algebra curriculum" per se, involves the development of a habit of mind that transcends the particular resource being used and allows elementary teachers to see opportunities for algebraic thinking, and functional thinking in particular, in the mathematics they already teach.

\subsection{Using children's functional thinking to leverage teacher learning}

Integrating functional thinking into instruction does not rest solely on the particular materials the teacher chooses or develops. It requires an "algebra sense" by which teachers can identify occasions in children's thinking to extend conversations about arithmetic to those that explore mathematical generality. While the task one chooses can certainly support this, teachers also need the skills to interpret what children are writing about and talking about. In turn, a written or verbal record of student thinking can serve as a tool to engage teachers in thinking about content and practice.

The work of Cognitively-Guided Instruction (Carpenter \& Fennema 1999) has been significant in bringing student thinking to the fore in how people conceptualize and engage in teacher professional development. More recently, researchers have extended this approach as a tool in the development of teachers' early algebraic thinking (Franke, Carpenter, Levi, \& Fennema 2001, Kaput \& Blanton to appear). The assumption is that focusing on children's (algebraic) thinking in professional development builds teachers' capacity to identify classroom opportunities for generalization and to understand the representational, linguistic and symbolic tools that support this and the particular ways students use these to reason algebraically. Thus, if teachers are to build generality into their instruction, they must become engaged in and by what students are saying, doing and writing as a catalyst for building their own classroom algebraic discourse, and they must be given occasions to use these classroom artifacts to negotiate mathematical and instructional knowledge within teacher communities of practice. As teachers think collectively about how children make sense of data, whether and how they attend to how quantities relate, the kinds of meaning they derive from tables and graphs, and how they use symbols in describing and reasoning with mathematical ideas, they have the potential to build functional thinking into instruction in deeper and more compelling ways (Kaput \& Blanton to appear). 


\subsection{Creating Classroom Culture and Practice to Support Functional Thinking}

Building on children's functional thinking in instruction requires that a culture of practice that promotes this type of thinking exists. Classrooms in which children's functional thinking can thrive are those in which the teacher has established socio-mathematical norms of conjecturing, arguing, and generalizing in purposeful ways, where the arguments are taken seriously by students as ways of building reliable knowledge. Robust functional thinking requires children to interact with complex mathematical ideas, to negotiate new notational systems and to understand and use representational tools as objects for mathematical reasoning. It requires that the teacher respect and encourage these processes as standard practice on a daily basis, not as occasional enrichment treated as separate from the "regular" work of learning and practicing arithmetic.

The teacher narratives included here illustrate the kinds of classroom practice and culture that can support the development of children's functional thinking. For example, the Growing Caterpillar narrative depicts ways of doing mathematics in which the teacher (1) followed students' thinking in shaping a lesson's the agenda ("I showed my students my caterpillar example and all I wanted them to see was how I developed the problem. I had no idea that they would begin to solve the problem. I couldn't stop them".), (2) placed the responsibility for conjecture, argumentation and justification with students ("Okay, what do you think the pattern is?", "How many of you agree with Jak?", "Why is that Meg?"), (3) cultivated children's use of representational structures as tools for reasoning (Meg: "I don't know. I have to do a tchart"), (4) encouraged the use of symbolic notational systems as valid forms of mathematical expression (Jak: "I think it is $x$ times 2 plus one"; Meg: "Because if it was $x$ times 2 plus 1 , then $x$ would be one and $y$ would be three. And, it's not. It's $x=1$ and $y=2$ "; Joe: "I see it, I know the formula!... It's $x \square x+1=y$ "), and (5) used children's utterances to craft an idea-building, dialogic discourse that led to symbolizing a functional relationship. In short, these aspects of practice allowed children to construct a mathematical generalization about the caterpillar's growth.

Children's role in this process is critical; we are not advocating a form of instruction in which children do not actively participate in the development of conjectures, the construction of arguments, the establishment of generalizations, or the use of notation, language, and tools for reasoning about functions. All of these experiences are critical components of the kind of classroom culture that makes functional thinking viable when it does occur.

\section{Conclusion}

This article elaborates the position that elementary school children are capable of functional thinking and that its study in the elementary grades can affect their success in mathematics in later grades. We propose that elementary grades mathematics extend beyond the fairly common focus on recursive patterning to include curriculum and instruction that deliberately attends to how two or more quantities vary in relation to each other and that begins to scaffold these notions from the start of formal schooling. Because there is a fundamental conceptual shift that must occur in how teachers and students attend to data in recursive patterning as opposed to covariational or correspondence relationships, we speculate that the emphasis on recursive patterning that does occur in elementary grades curricula, could, if taught in isolation, impede the development of covariational and correspondence thinking about functions in later grades.

Children's capacity for functional thinking raises the issue of how it might be nurtured by curriculum and instruction in the elementary grades. We advocate here a habit of mind, not just curricular materials, whereby teachers understand how to transform and extend their current resources so that the mostly arithmetic content can become opportunities for pattern building, conjecturing, generalizing, and justifying mathematical relationships and how to embed this mathematics within the kinds of socio-mathematical norms that allow children to build mathematical generality. Generalizing is a human activity and an innate, natural capacity that young children bring to the classroom (Mason to appear). Curriculum and instruction should build on these natural abilities to provide a deeper, more compelling mathematical experience for young children.

\section{References}

Behr, M.J.; Lesh, R.; Post, T.R.; \& Silver, E.A. (1983): Rational-number concepts. - In: R. Lesh \& M. Landau (Eds.), Acquisition of mathematics concepts and processes. New York: Academic Press, p. 91-126

Blanton, M. (to appear): Elementary school children can do algebra and even enjoy it. - In: B. Dougherty \& L. Lee (Eds.), The algebra lesson: What we have learned through classroom research. Math Education Research Series: Information Age

Blanton, M.; \& Kaput, J. (2000): Generalizing and progressively formalizing in a third grade mathematics classroom: Conversations about even and odd numbers. In: M. Fernández (Ed.) Proceedings of the Twenty-Second Annual Meeting of the North American Chapter of the International Group for the Psychology of Mathematics Education, Columbus, OH, ERIC Clearinghouse, p.115-119

Blanton, M.; \& Kaput, J. (2003): Developing elementary teachers' algebra eyes and ears. - In: Teaching Children Mathematics, Vol. 10(2), p. 70-77

Blanton, M.; \& Kaput, J. (2004): Elementary grades students' capacity for functional thinking. - In: Proceedings of the International Group for the Psychology of Mathematics Education, Bergen, Norway: Bergen University College, Vol, 2, p. 135-142

Blanton, M.; \& Kaput, J. (to appear): Characterizing a classroom practice that promotes algebraic reasoning. Journal for Research in Mathematics Education.

Boyer, C. (1946): Proportion, equation, function: Three steps in the development of a concept. - In: Scripta Mathematica, Vol. 12, p. 5-13

Brizuela, B.; Carraher, D.W.; \& Schliemann, A.D. (2000): Mathematical notation to support and further reasoning ("to help me think of something"). Paper presented at the NCTM Research Pre-Session

Brizuela, B.; \& Earnest, D. (to appear): Multiple notational systems and algebraic understandings: The case of the "Best 
Deal" problem. - In: J. Kaput, D. Carraher, \& M. Blanton (Eds.), Algebra in the early grades. Mahwah, NJ: Lawrence Erlbaum Associates

Brizuela, B.; \& Schliemann A. (2003): Fourth-graders solving equations. - In: N. Pateman, B. Dougherty, \& J. Zilliox (Eds.), Proceedings of the 2003 Joint Meeting of PME and PME-NA, Honolulu, HI: College of Education, Vol. 2, p. 137-143

Carpenter, T.P.; \& Fennema, E., (1999): Children's mathematics: Cognitively guided instruction. Portsmouth, NH: Heinemann

Carpenter, T.; Franke, M.; \& Levi, L. (2003): Thinking mathematically: Integrating arithmetic and algebra in elementary school. Portsmouth, NH: Heinemann

Carraher, D.W.; Schliemann, A.D.; \& Brizuela, B. (2000): Early Algebra, Early Arithmetic: Treating operations as functions. - In: In: M. Fernández (Ed.) Proceedings of the Twenty-Second Annual Meeting of the North American Chapter of the International Group for the Psychology of Mathematics Education, Columbus, OH, ERIC Clearinghouse

Confrey, J.; \& Smith, E. (1991): A framework for functions: Prototypes, multiple representations, and transformations. In: R. G. Underhill (Ed.), Proceedings of the 13th Annual Meeting of the North American Chapter of the International Group for the Psychology of Mathematics Education, Blacksburg, VA, Vol. 1, p. 57-63

Franke, M.L.; Carpenter, T.P.; Levi, L.; \& Fennema, E. (2001): Capturing teachers' generative growth: A follow-up study of professional development in mathematics. - In: American Educational Research Journal, Vol. 38(3), p. 653-690

Freudenthal, H. (1982): Variables and functions. - In: G. V. Barneveld \& H. Krabbendam (Eds.), Proceedings of conference on functions, Enschede, The Netherlands: National Institute for Curriculum Development, p. 7-20

Goldin, G.; \& Shteingold, N. (2001): Systems of representations and the development of mathematical concepts. - In: A. A. Cuoco \& F. R. Curcio (Eds.), The roles of representation in school mathematics. NCTM 2001 Yearbook. Reston, Virginia: National Council of Teachers of Mathematics, p. 1-23

Hamley, H.R. (1934): Relational and functional thinking in mathematics. - In: The 9th Yearbook of NCTM. New York: Bureau of Publications, Teachers College, Columbia University

Kaput, J. (1995): A research base for algebra reform: Does one exist? - In: D. Owens, M. Reed, \& G. M. Millsaps (Eds.), Proceedings of the 17th Annual Meeting of the North American Chapter of the International Group for the Psychology of Mathematics Education, Columbus, OH: The ERIC Clearinghouse for Science, Mathematics, and Environmental Education, Vol. 1, p. 71-94

Kaput, J. (1999): Teaching and learning a new algebra. - In: E. Fennema, \& T. Romberg (Eds.), Mathematics classrooms that promote understanding. Mahwah, NJ: Lawrence Erlbaum Associates, p. 133-155

Kaput, J.; \& Blanton, M. (2001). Student achievement in algebraic thinking: A comparison of third-graders' performance on a state fourth-grade assessment. - In: R. Speiser, C. Maher, \& C. Walter (Eds.), Proceedings of the 23rd Annual Meeting of the North American Chapter of the Psychology of Mathematics Education, Vol. 1, p. 99-108.

Kaput, J.; \& Blanton, M. (to appear): Algebrafying the elementary mathematics experience in a teacher-centered, systemic way. - In: T. A. Romberg, T. P. Carpenter, \& F. Dremock (Eds.), Understanding mathematics and science matters. Mahwah, NJ: Lawrence Erlbaum Associates

Lins, R.; \& Kaput, J. (2004): The early development of algebraic reasoning: The current state of the field. - In: H.
Chick, K. Stacy (Eds.), The future of the teaching and learning of algebra: The 12th ICMI Study. London: Kluwer

Mason, J. (to appear): Making use of children's powers to produce algebraic thinking. - In: J. Kaput, D. Carraher, \& M. Blanton (Eds.), Algebra in the early grades. Mahwah, NJ: Lawrence Erlbaum Associates

National Council of Teachers of Mathematics. (2000): Principles and standards for school mathematics. Reston, VA: Author

National Research Council (2001): Adding it up: Helping children learn mathematics. Washington, DC: National Academy Press

Romberg, T.; \& Kaput, J. (1999): Mathematics worth teaching, mathematics worth understanding. - In: E. Fennema, \& T. Romberg (Eds.), Mathematics classrooms that promote understanding. Mahwah, NJ: Lawrence Erlbaum Associates, p. 3-32

Schliemann, A.D.; \& Carraher, D.W. (2002): The Evolution of Mathematical Understanding: Everyday Versus Idealized Reasoning. - In: Developmental Review, Vol. 22(2), p. 242266

Schliemann, A.D.; Carraher, D.W.; \& Brizuela, B. (2001): When tables become function tables. - In: M. HeuvelPanhuizen (Ed.), Proceedings of the Twenty-fifth International Conference for the Psychology of Mathematics Education, Utrecht, The Netherlands, Vol. 4, p. $145-152$

Schwartz, J. (1990): Getting students to function in and with algebra. - In: G. Harel \& E. Dubinsky (Eds.), The concept of function: Aspects of epistemology and pedagogy. Washington, DC: Mathematics Associations of America, p. 261-289

Smith, E. (2003): Stasis and change: Integrating patterns, functions, and algebra throughout the K-12 curriculum. - In: J. Kilpatrick, W. G. Martin, \& D. Schifter (Eds), A research companion to principles and standards of school mathematics. Reston, VA: National Council of Teachers of Mathematics, p. 136-150

Smith, E. (to appear): Representational thinking as a framework for introducing functions in the elementary curriculum. - In: J. Kaput, D. Carraher, \& M. Blanton (Eds.), Algebra in the early grades. Mahwah, NJ: Lawrence Erlbaum Associates

Thompson, A.G.; Philipp, R.A.; Thompson, P.W.; \& Boyd, B.A. (1994): Calculational and conceptual orientations in teaching mathematics. - In: A. Coxford (Ed.), 1994 Yearbook of the NCTM. Reston, VA: NCTM, p. 79-92

Vygotsky, L. (1962): Thought and language. (E. Hanfmann and G. Vakar, Trans.). Cambridge, MA: Massachusetts Institute of Technology. (Original work published in 1934.)

\section{Authors}

Blanton, M., Dr., Mathematics Department, University of Massachusetts Dartmouth, 285 Old Westport Road, No. Dartmouth, MA, 02747-2300, USA.

E-mail: mblanton@umassd.edu

Kaput, J., Dr., Mathematics Department, University of Massachusetts Dartmouth, 285 Old Westport Road, No. Dartmouth, MA, 02747-2300, USA. E-mail: jkaput@umassd.edu. 